\title{
Geometrical Interpretation of a few concomitants of the cubic in the Argand Plane.
}

By W. SAdDler.

(Received 17th January 1924. Read 9th June 1923).

\$1. Let $f=a_{2}^{3}=a_{2}^{\prime 8}=a_{0} z^{3}+3 a_{1} z^{2}+3 a_{2} z+a_{3}=0$

be the binary cubic whose coefficients $a_{i}$ are complex numbers represented on the Argand Plane. Then if its roots are $z_{1}, z_{2}, z_{3}$, the three corresponding points form the vertices of a triangle $A_{1} A_{2} A_{3}$. Let this triad of points be said to represent the cubic. Then its Hessian

$$
H=\left(a a^{\prime}\right)^{2} a_{\mathrm{z}} a_{\mathrm{z}}^{\prime}
$$

is represented by a certain pair of other points; likewise every first polar

$$
\left(y \frac{\partial}{\partial z}\right) f=a_{z}^{2} a_{y}
$$

associates a definite pair of points $(z)$ with any given point $(y)$. It is understood that $y, z \ldots$ are each complex numbers.

In particular the first polar

$$
\frac{d f}{d z} \text { i.e. } a_{0} z^{2}+2 a_{1} z+a_{2}
$$

of the point at infinity gives, as is well known, the real foci of the conic, which touches at their mid points the lines joining $A_{1} A_{2} A_{3}$. The centre of the conic is the point $-\frac{a_{1}}{a_{0}}$.

Since $(f, H)^{2}=0$ identically $y^{*}$ then $H$ is apolar to the first polar of any point $y$.

Now the mid point $w$ of the two points $z$ represented by (3) whose equation - non-symbolically-is

is given by

$$
\left(a_{0} y+a_{3}\right) z^{2}+2\left(a_{1} y+a_{2}\right) z+a_{2} y+a_{3}=0
$$

$$
w=-\frac{a_{1} y+a_{3}}{a_{0} y+a_{1}} \text { i.e. } a_{0} y w+a_{1}(y+w)+a_{2}=0 .
$$

" $C f$. Grace and Young, Algebra of Invariants (190) p. 
Thus $y, w$, form an involution whose double points are

$$
a_{0} z^{2}+2 a_{1} z+a_{2}=0 \text {. }
$$

Hence tho points $y, w$, are harmonic to the focal points (4) ..........I.

$\S 2$. This gives us a Geometrical construction for the roots of the first polar of the point $y$.

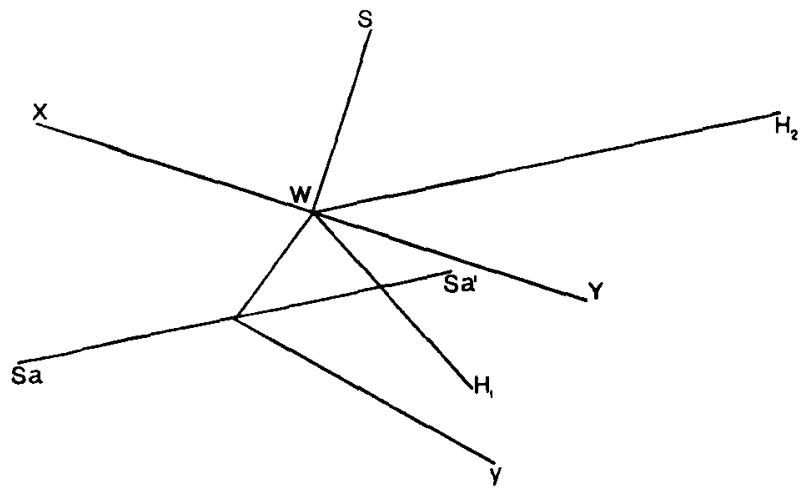

Fig. 1

(The figure is not drawn accurately to scale.)

Let $S_{a}$ and $S_{a^{\prime}}$ be the focal points and $y$ the given point. Determine $W$ so that $\left(S_{a} S_{a^{\prime}}, W y\right)$ are apolar; in fact $W$ is the harmonic conjugate of $y$ with regard to $S_{a}, S_{a^{\prime}}$ on the circle $S_{a} S_{a^{\prime}} y$. Let $H_{1} H_{2}$ be the Hessian points. ${ }^{*}$ The Geometrical determination of these points will be explained below. Let $X Y$ bisect the angle $H_{1} W H_{2}$. Take $S$ the point where the perpendicular to $X Y$ at $W$ and the perpendicular to $H_{1} H_{2}$ at its mid point meet: then the circle whose centre is $S$ and radius $S H_{1}$ will meet the line $X Y$ at the points $X Y$ which represent the first polar of $y$.

For $W X, W Y=W H_{2}, W H_{1}$ and the line $X Y$ bisects the angle $H_{2} W H_{1}$.

The point $y$ together with the second polar of $y$ with regard to $f$, i.e. $a_{y}^{2} a_{z}=0$ are apolar to the first polar of $y$ since

$$
\text { (a } \left.a^{\prime}\right) a_{y}^{2} a_{y}^{\prime 2}=0 \text {. }
$$

Hence we can also construct geometrically the second polar of $y$, and incidentally the apolar triad $a_{x} a_{y} a_{z}=0$.

\footnotetext{
* See Grace and Young, loc. cit., pp. 209, 211.
} 
\$3. If $A_{1} A_{2} A_{3}$ represent roots of $a_{z}^{3}=0$ the first polar of $A_{1}$ is $A_{1}$ itself together with the point $P_{1}$ which is the fourth harmonic with regard to $A_{2} A_{3}$ on the circle $A_{1} A_{2} A_{3}$ : i.e. $P_{1}$ is one of the roots of the cubic covariant of $a_{z}^{3}=0$.

This gives us immediately the algebraical expression for the roots of the cubic covariant in terms of those of the equation $a_{z}^{3}=0$.

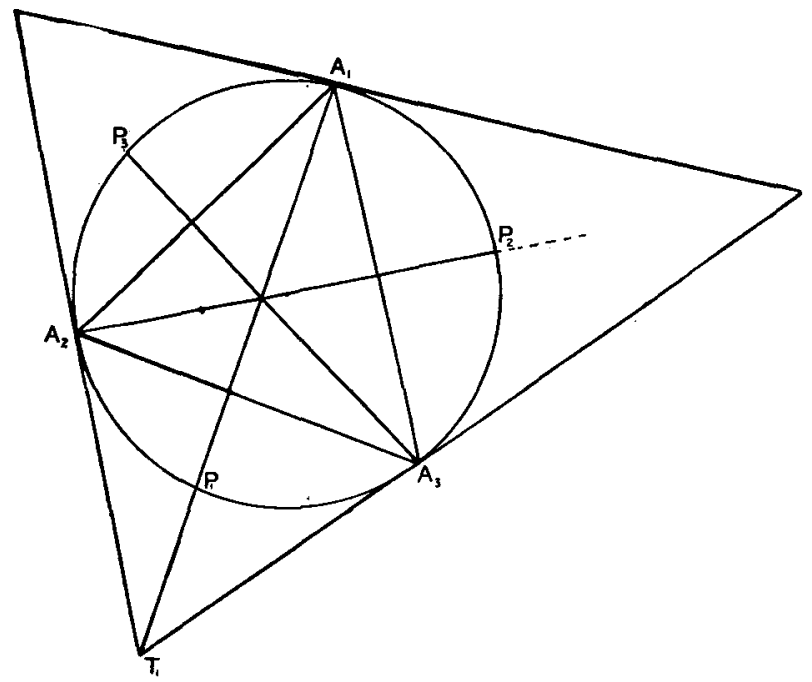

Fig. 2

Tangents are drawn to the circle circumscribing $A_{1} A_{2} A_{3} . A_{1}$ is joined to $T_{1}$ where the tangents at $A_{2}$ and $A_{3}$ meet. The line $A_{1} T_{1}$ cuts the circle in $P_{1}$.

The Hessian points * are got by drawing a circle through $A_{1}$ so that $A_{2} A_{3}$ are inverse points with regard to this circle and a second circle through $A_{2}$ so that $A_{1} A_{3}$ are inverse points with respect to it: these two circles intersect in the Hessian points. These circles also pass respectively through $P_{1}$ and $P_{2}$ since $A_{1} P_{1}$ being the first polar of the point $A_{1}$ are apolar to the Hessian points.

The point $A_{1}$ and the mid point of $A_{1} P_{1}$ are, by result $\mathrm{I}$. harmonic to the focal points.

- Cf. Grave and Young, loc. cit. 
Similarly by considering the cubic covariant in place of the original cubic we can prove that the point $P_{1}$ and the mid point of $A_{1} P_{1}$ are harmonic to the focal points of a similar conic inscribed in the triangle $P_{1} P_{2} P_{3}$.

So the centres of these two conics are harmonic to the points $H_{1} H_{2}$, and also the pairs of real foci of these conics are also harmonically related as may be easily verified.

\$4. Two Cubics. The apolar invariant of the two cubics $f=a_{z}^{3}=0$ and $f^{\prime}=b_{z}^{3}=0$ is $(a b)^{3}=0$. The foci being the first polar of the point at infinity: let this point be $\rho$. Then $a_{\rho} a_{z}^{2}=0$, $b_{p} b_{z}^{2}=0$ give the four foci $S_{a}, S_{a^{\prime}} ; S_{b}, S_{b^{\prime}}$.
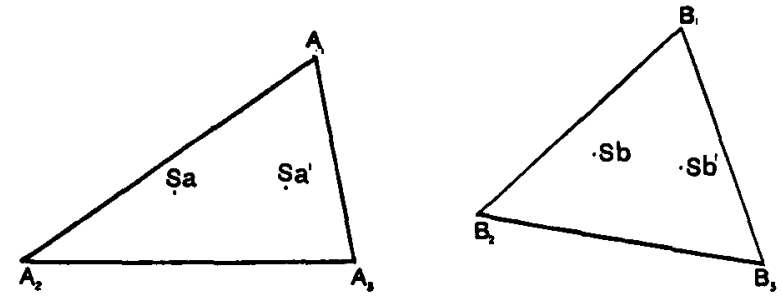

Fig. 3

Choose $y$ so that its first polar with respect to $f^{\prime}=0$, i.e. $b_{y} b_{a}^{2}=0$ is apolar to $S_{a} S_{a^{\prime}}$, therefore $b_{y} a_{\rho}(a b)^{2}=0$

This is a homography between $y$ and $\rho$. The double points are points $K K^{\prime}$ given by $(a b)^{2} a_{\kappa} b_{\kappa}=0$, i.e. by the vanishing of the second transvectant of the two cubics.

If (5) is an involution $y$ and $\rho$ can be interchanged therefore or

$$
\begin{aligned}
& (a b)^{2} a_{\rho} b_{y}-(a b)^{2} a_{y} b_{\rho}=0, \\
& (a b)^{3}(y \rho)=0 .
\end{aligned}
$$

Thus unless $y=\rho$ which only happens when these two points coincide at $K$ or $K^{\prime},(5)$ is an involution if $(a b)^{3}$ vanishes and if so (5) holds for all points of the plane.

\$5. The last result may be shown non-symbolically.

For the first polar of $y$ with regard to $f$ is

$$
\left(a_{0} y+a_{1}\right) z^{2}+2\left(a_{1} y+a_{2}\right) z+\left(a_{2} y+a_{3}\right)=0
$$

and of $w$ with regard to $f^{\prime}$ is

$$
\left(b_{0} w+b_{1}\right) z^{2}+2\left(b_{1} w+b_{2}\right) z+\left(b_{2} w+b_{3}\right)=0 .
$$


These are apolar if

$$
\begin{aligned}
y w\left(a_{0} b_{2}+\right. & \left.a_{2} b_{0}-2 a_{1} b_{1}\right)+y\left(b_{1} a_{2}+a_{0} b_{3}-2 a_{1} b_{2}\right) \\
& +w\left(a_{1} b_{2}+a_{3} b_{0}-2 a_{2} b_{1}\right)+a_{3} b_{1}+a_{1} b_{3}-2 a_{2} b_{2}=0 .
\end{aligned}
$$

This is an involution whose double points are the roots of the second transvectant

$$
\begin{aligned}
& \left(a_{0} b_{3}+a_{2} b_{0}-2 a_{1} b_{1}\right) z^{2}+\left(a_{0} b_{3}+a_{3} b_{0}-a_{1} b_{2}-a_{2} b_{1}\right) z \\
& +\left(a_{1} b_{3}+a_{3} b_{1}-2 a_{2} b_{3}\right)=0 \\
& \text { if } \quad a_{0} b_{3}-3 a_{1} b_{2}+3 a_{2} b_{1}-a_{3} b_{0}=0 \text {, } \\
& \text { i.e. } \quad(a b)^{3}=0 \text {. }
\end{aligned}
$$

\$6. The first polars of the origin with respect to $f$ and $f^{\prime}$ are

$$
\begin{aligned}
& a_{1} z^{2}+2 a_{22} z+a_{3}=0, \\
& b_{1} z^{2}+2 b_{2} z+b_{3}=0 .
\end{aligned}
$$

Now (6) is apolar to the "focal" points of $f$, and (7) is apolar to the " focal "points of $f$ if

$$
\begin{aligned}
& \left(a_{1} b_{1}-a_{2} b_{0}\right) z^{2}+\left(a_{1} b_{2}-a_{3} b_{0}\right) z+\left(a_{2} b_{2}-a_{3} b_{1}\right)=0, \\
& \left(a_{1} b_{1}-a_{0} b_{2}\right) z^{2}+\left(b_{1} a_{2}-a_{0} b_{3}\right) z+\left(a_{2} b_{2}-a_{1} b_{3}\right)=0 .
\end{aligned}
$$

But the second transvectant is the sum of (8) and (9). Hence the pair of points apolar to $(8)$ and $(9)$ is apolar to the second transvectant

The centres of the conics for the two cubics, i.e. the points $-\frac{a_{1}}{a_{0}},-\frac{b_{1}}{b_{0}}$ have for their first polars respectively

and

$$
\left(b_{0} a_{1}-a_{0} b_{1}\right) z^{2}+2 z\left(b_{1} a_{1}-b_{2} a_{0}\right)+b_{2} a_{1}-b_{3} a_{0}=0
$$

$$
\left(a_{0} b_{1}-a_{1} b_{0}\right) z^{2}+2 z\left(b_{1} a_{1}-a_{2} b_{0}\right)+a_{2} b_{1}-a_{3} b_{0}=0 \text {. }
$$

The mid point of the pair of points apolar to these two can be shown to be the mid point of the pair of points which are the roots of the second transvectant, $\left(a_{z}^{3}, b_{z}^{3}\right)^{2}$.

This enables us to construct the points representing this second transvectant. 